\title{
REVIEW
}

\section{Transarterial Radioembolization with Yttrium-90 for the Treatment of Hepatocellular Carcinoma}

\author{
Joseph Ralph Kallini · Ahmed Gabr · Riad Salem • Robert J. Lewandowski
}

Received: February 18, 2016/Published online: April 2, 2016

(C) The Author(s) 2016. This article is published with open access at Springerlink.com

\begin{abstract}
Background: Hepatocellular carcinoma (HCC) is a common cause of worldwide mortality. Transarterial radioembolization (TARE) with yttrium-90 (Y90), a transcatheter intra-arterial procedure performed by interventional radiology, has become widely utilized in managing HCC.
\end{abstract}

Methods: The following is a focused review of TARE covering its commercially available

Enhanced content To view enhanced content for this article go to http://www.medengine.com/Redeem/9B84 F06014B881BF.

J. R. Kallini · A. Gabr · R. Salem .

R. J. Lewandowski $(\bowtie)$

Department of Radiology, Section of Interventional Radiology, Northwestern Memorial Hospital, Robert

H. Lurie Comprehensive Cancer Center, Chicago,

IL, USA

e-mail: r-lewandowski@northwestern.edu

R. Salem

Division of Transplantation, Department of Surgery,

Comprehensive Transplant Center, Northwestern

University, Chicago, IL, USA

R. Salem

Division of Hematology and Oncology, Department of Medicine, Northwestern University, Robert $\mathrm{H}$. Lurie Comprehensive Cancer Center, Chicago, IL, USA products, clinical considerations of treatment, salient clinical trial data establishing its utility, and the current and future roles of TARE in the management of HCC.

Results: TARE is indicated for patients with unresectable, intermediate stage HCC. The two available products are glass and resin microspheres. All patients undergoing TARE must be assessed with a history, physical examination, clinical laboratory tests, imaging, and arteriography with macroaggregated albumin. TARE is safe and effective in the treatment of unresectable HCC, as it has a safer toxicity profile than chemoembolization, longer time-to-progression, greater ability to downsize and/or bridge patients to liver transplant, and utility in tumor complicated by portal vein thrombosis. TARE can also serve as an alternative to ablation and chemotherapy. Conclusion: TARE assumes an integral role in the management of unresectable HCC and has been validated by numerous studies.

Keywords: Hepatocellular carcinoma (HCC); Oncology; Radioembolization; Transarterial radioembolization (TARE); Yttrium-90 (Y90) 


\section{INTRODUCTION}

Transarterial radioembolization (TARE) is a transcatheter intra-arterial procedure performed by the interventional radiologist for the treatment of primary and secondary hepatic cancers. Microspheres impregnated with the radioisotope yttrium-90 $\left(\mathrm{Y90},{ }^{90} \mathrm{Y}\right)$ are selectively delivered through the hepatic vasculature to the target tumor(s). Selective intra-arterial injection of these microspheres allows for the safe administration of high radiation doses to the tumor. While transarterial chemoembolization (TACE) is the standard treatment paradigm for patients with unresectable intermediate stage-Barcelona Clinic Liver Cancer (BCLC) stage Bhepatocellular carcinoma (HCC), evidence supports an increasing role for TARE. While imaging response rates and median overall survival from day of treatment appear comparable between TACE and TARE, most patients treated with TARE have more advanced disease than those with TACE and the treatment is less selective. Despite this, TARE exceeds TACE in terms of time-to-progression (TTP) of disease, toxicity profile, and post-treatment quality of life. TARE also has demonstrated utility in patients with portal vein thrombosis (PVT), a relative contraindication to TACE. TARE is also useful as an alternative to ablation and in facilitating resection of BCLC stage A tumors. This review discusses the salient features of TARE, landmark clinical studies establishing its role in directed cancer therapy, and future directions. This article does not contain any studies with human participants or animals performed by any of the authors.

\section{OVERVIEW OF HEPATOCELLULAR CARCINOMA}

\section{Synopsis}

Hepatic cancer is the fifth most frequently diagnosed cancer in men and seventh in women worldwide, HCC being the most common. Incidence rates of HCC are increasing across the globe as a result of intravenous drug abuse, hepatitis $\mathrm{C}$ virus (HCV) infection, and nonalcoholic steatohepatitis (NASH) [1]. The incidence of HCC in the USA is approximately 3 per 100,000 persons, with significant gender, ethnic, and geographic variations. Approximately 22,000 new cases and 18,000 deaths occur in the USA yearly [2].

From $75 \%$ to $85 \%$ of HCC is associated with underlying cirrhosis. Several underlying risk factors are associated with HCC: hepatitis B virus (HBV) infection, HCV infection, alcohol ingestion, autoimmune hepatitis, and NASH. Less commonly associated are primary biliary cirrhosis and metabolic syndromes (hemochromatosis, Wilson's disease, $\alpha_{1}$-antitrypsin deficiency) [2].

The clinical presentation of HCC includes nausea, abdominal pain, weight loss, abdominal fullness, and jaundice. Hematemesis results from esophageal variceal bleeding secondary to underlying portal hypertension. On physical examination, $50-90 \%$ of patients have hepatomegaly due to associated liver disease. Ascites presents in $30-60 \%$ of patients. Abdominal bruits are noted in 6-25\%. Splenomegaly is a common finding, mainly due to portal hypertension. Other signs of chronic liver disease presenting with HCC 
include jaundice, abdominal vein dilatation, palmar erythema, hepatomegaly, gynecomastia, testicular atrophy, and peripheral edema. Budd-Chiari syndrome results from HCC invasion of the hepatic veins, suspected by tense ascites and pain on palpation of the liver [2].

\section{Current Management}

The BCLC system is currently the most commonly used staging system to guide the management of HCC (Fig. 1) [3]. TACE was established as the standard of care therapy for unresectable intermediate stage HCC (BCLC B) in 2002 on the basis of two prospective randomized clinical trials versus best supportive care $[4,5]$. TARE has been typically employed in patients with unresectable HCC deemed not to be good candidates for TACE or those who have failed prior TACE procedures. While TACE is most often delivered in a segmental or selective fashion, TARE has been historically delivered in a lobar or whole liver manner. Thus, much of the existing evidence for TARE for patients with HCC was produced in patients with more advanced disease than that for TACE $[6,7]$.

According to the National Cancer Center Network (NCCN), locoregional therapies (TACE and TARE) have an established role in neoadjuvant treatment of HCC if the waiting

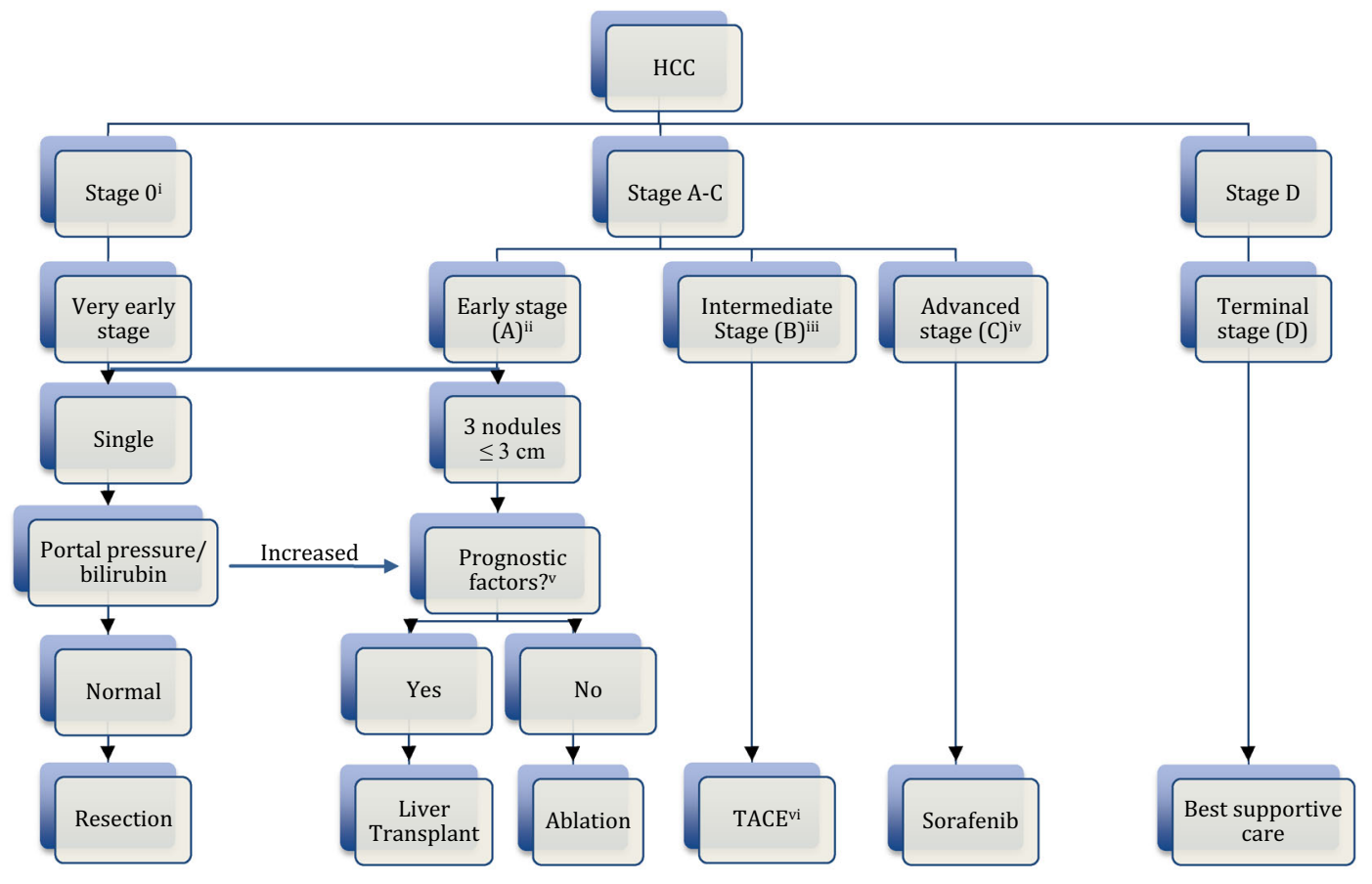

Fig. 1 BCLC staging system and treatment strategy (adapted from [3]). $i$ Very early stage 0 : single lesion $<2 \mathrm{~cm}$, carcinoma in situ. ii Early stage A: single or 3 nodules $\leq 3 \mathrm{~cm}$. iii Intermediate stage B: multinodular. iv Advanced stage C: portal invasion, $\geq 1$ involved lymph node, or metastasis. $v$ Prognostic factors include variables mostly related to HCC that are defined as the "Milan criteria": (single tumors less than $5 \mathrm{~cm}$ or 3 nodules less than $3 \mathrm{~cm}$ ). Whether patients at stage 0 can be offered local ablation as a first-line treatment option is a topic of controversy since transplantation is potentially curative [3]. vi TACE transarterial chemoembolization 
list times for transplant exceed 6 months (evidence 2D; recommendation 2B) [3]. The NCCN guidelines recognize the role of intra-arterial liver-directed therapy for the management of HCC. All such therapies, including TARE, are contraindicated with serum bilirubin greater than $3 \mathrm{mg} / \mathrm{dL}$ unless segmental injection can be performed. Such therapies are relatively contraindicated in main PVT and Child-Pugh C disease, although TARE has proven benefit in main and branch PVT [8]. Treatment allocation should be decided by a multidisciplinary team of hepatologists, pathologists, radiologists, liver surgeons, and oncologists guided by personalized-based medicine [9].

\section{TRANSARTERIAL RADIOEMBOLIZATION}

HCC receives approximately $90 \%$ of its blood supply from the hepatic artery, while the normal liver parenchyma obtains $70 \%$ of its supply from the portal venous system. TARE capitalizes on this concept by delivering targeted therapy to HCC with minimal parenchymal compromise [10]. Y90 is the most commonly used radioactive element in radioembolization. The unstable Y90 radioisotope undergoes logarithmic beta decay into the stable element zirconium-90 at a half-life of 2.67 days $(64.2 \mathrm{~h})$. In doing so, it emits a high-speed electron, known as a beta particle, which induces direct cytotoxic destruction to the target tumor. Tissue penetration of Y90 ranges from 2.5 to $11 \mathrm{~mm}$. A radiation dose of up to $170 \mathrm{~Gy}$ can be administered [11].

Patients with unresectable HCC and a life expectancy of at least 3 months are considered eligible candidates for TARE; patients with excessive tumor burden and limited hepatic function are ineligible [12]. Adverse events reported with TARE include fatigue, nausea, abdominal pain, gastrointestinal ulcers, and transaminitis [13-20]. Unlike TACE, its transarterial counterpart TARE is a predominantly outpatient procedure [21].

Currently, two Y90 products are commercially available: TheraSphere $^{\circledR}$ glass microspheres (BTG, Canada) and SIR-Spheres ${ }^{\circledR}$ resin microspheres (Sirtex Medical, Woburn, MA, USA).

\section{Glass Microspheres}

The insoluble, Y90-impregnated glass microspheres have a diameter of $20-30 \mu \mathrm{m}$ and an activity of $2500 \mathrm{~Bq}$ per sphere at the time of calibration. Glass microspheres are indicated for inoperable HCC and HCC complicated by PVT, with approval by the US Food and Drug Administration (FDA) under a Humanitarian Device Exemption (based on proven safety and potential clinical benefit) [22]. A total of 1.2 million microspheres produce $3 \mathrm{GBq}$ of activity (2500 Bq per sphere).

\section{Resin Microspheres}

Resin microspheres received full premarketing FDA approval in 2002 for unresectable colorectal metastases in conjunction with intrahepatic floxuridine (FUDR) chemotherapy [23]. Resin microspheres consist of biocompatible resin-based microspheres with a diameter of $20-60 \mu \mathrm{m}$ and an activity of $50 \mathrm{~Bq}$ per sphere [10]. Resin microspheres have a lower density of Y90 per sphere than glass microspheres, meaning more spheres are required to administer a given dose, and thus there is a 
higher embolic effect with a given administration [24]. A total of 40-80 million resin microspheres produce $3 \mathrm{GBq}$ of activity (50 Bq per sphere), as opposed to 1.2 million glass microspheres.

\section{CLINICAL CONSIDERATIONS}

\section{Patient Evaluation}

Patient selection for TARE involves an assessment of disease burden, biochemical profile, and performance status. All patients undergoing TARE require

1. History and physical examination, including Eastern Cooperative Oncology Group (ECOG) performance status assessment

2. Clinical laboratory tests

3. Imaging

4. Arteriography/macroaggregated albumin (MAA) lung shunting study

Patients with an ECOG score greater than 2individuals who are unable to ambulate for more than half of the day-are excluded. Laboratory workup should include a hepatic panel and serum $\alpha$-fetoprotein (AFP). Patients should have a bilirubin less than or equal to $2 \mathrm{mg} / \mathrm{dL}$, although the treating physician can consider selective therapy. Required imaging includes chest computed tomography (CT), either a CT or magnetic resonance (MR) scan of the abdomen, and bone scan [10]. Ideal candidates have HCC confined to the liver with tumor comprising less than $70 \%$ of the liver volume. Patients who have had a prior intervention of the biliary tree may require prophylactic antibiotics prior to TARE.

For patients with a solitary tumor and normal serum bilirubin, Y90 can be administered via a lobar or segmental injection. For patients with solitary HCC but elevated bilirubin, TARE should only be performed segmentally, which concentrates the Y90 to the tumor and spares the most uninvolved liver. For patients with multiple tumors and/or bilobar HCC and normal serum bilirubin, sequential lobar treatments are performed. Patients with abnormal bilirubin and multinodular/bilobar disease are generally excluded.

\section{Pretreatment Angiography}

TARE requires pretreatment aortic, superior mesenteric, and celiac trunk angiography to accurately assess the hepatic vasculature, surrounding structures, portal vein patency, and the presence of arterioportal shunting [25]. HCC may parasitize blood flow from surrounding vessels; failure to recognize a vessel supplying the tumor may lead to incomplete treatment and/or treatment failure. In addition, angiography determines the presence of extrahepatic blood flow to other organs; microspheres injected through these nontarget vessels may result in adverse events. Coil embolization of nontarget vessels may be performed but is no longer routinely recommended [26].

\section{${ }^{99 m}$ Technetium Macroaggregated Albumin Nuclear Scan}

In the ${ }^{99 \mathrm{~m}}$ Tc-MAA nuclear scan, $4-5 \mathrm{mCi}$ of ${ }^{99 \mathrm{~m}}$ Tc-MAA is injected into the hepatic artery to assess splanchnic and pulmonary shunting. This test is performed to discern whether arteriovenous connections surrounding the tumor are diverting blood flow to the lungs; HCC is associated with a relatively high incidence of direct arteriovenous shunts that bypass the tumor capillary bed. The 
administration of microspheres could thus enter the lungs and cause radiation pneumonitis at sufficient doses [27]. From the MAA, the lung shunt fraction (LSF) is determined, which is defined as

LSF $=($ total lung counts $) /($ total lung counts

+ total abdomen counts).

A lung dose greater than 30 Gy per treatment or cumulative lung dose of $50 \mathrm{~Gy}$ places the patient at increased risk of radiation pneumonitis after radioembolization [10]. It is important to correlate the findings of angiography to the findings of the ${ }^{99 m}$ Tc-MAA scan. The preprocedure angiogram, $\quad{ }^{99 m}$ Tc-MAA scan, and radioembolization can all be performed on the same day in an outpatient setting in appropriately selected patients [21].

\section{Dose Calculation}

Dose calculation for glass microspheres utilizes three-dimensional reconstruction of the site encompassing the tumor in question in order to find the volume to be treated. The resultant tissue volume determined by CT is converted to tissue mass using the density conversion factor $1.03 \mathrm{~kg} / \mathrm{ml}$ [10]. The activity $(A)$ in $\mathrm{GBq}$ to be administered to the target area, assuming uniform distribution of microspheres, is expressed as follows:

$A=D \times m / 50$

where $D$ is the dose administered in Gy, and $m$ is the treated tissue mass in $\mathrm{kg}$. This equation assumes a uniform distribution of glass microspheres throughout the liver and complete in situ decay of the administered Y90. The goal of therapy for glass microspheres is to deliver a radiation dose of 120 Gy to the injected liver volume.

Many dose calculation methods for resin microspheres exist. An injected liver volume of $120 \mathrm{~Gy}$ to the tumor compartment in question is recommended and 50-70 Gy to the healthy liver, though this depends on the clinical scenario. The most commonly used is the body surface area (BSA) method, which is best suited for poorly delineated tumors and is represented by the formula

$A=\mathrm{BSA}-0.2+(\%$ Tumor burden $/ 100)$

where $A$ is the activity in GBq, BSA is the body surface area in square meters, and \% tumor burden is the percentage of the liver involved by tumor. This model of dosimetry for resin microspheres is based on whole-liver infusion; the calculated activity given to the entire liver is multiplied by the percentage of the target site as a proportion of the whole liver. The administered activity depends on percentage involvement by the tumor in the liver as calculated by a chosen model.

\section{Administration}

Once a patient is deemed eligible, TARE is performed on an outpatient basis either on a different day or in the same session [21]. Selective catheterization of the vessel chosen by pretreatment angiography is performed.

For glass microspheres, the final total dose administered is calculated by using the inverse of the dose formula:

$D=A \times 50 / \mathrm{m}$.

However, this formula needs to be corrected for two post-treatment confounding factors: residual activity $(R)$ not fully administered and lung shunt fraction (LSF): 
$D=A \times 50 \times(1-\mathrm{LSF}) \times(1-R) / \mathrm{m}$.

For resin microspheres, the administered activity depends on percentage of the liver involved by the tumor. Greater than 50\% requires $3.0 \mathrm{GBq}, 25-50 \%$ requires $2.5 \mathrm{GBq}$, and less than $25 \%$ requires $2.0 \mathrm{GBq}$. The dose is reduced depending on the degree of lung shunting as calculated by the LSF. LSF less than $10 \%$ requires no dose reduction. LSF between $10 \%$ and $15 \%$ requires a $20 \%$ dose reduction. LSF between $15 \%$ and $20 \%$ requires a $40 \%$ dose reduction. LSF greater than $20 \%$ cannot be treated [23].

\section{Post-Treatment Assessment}

The tumor response of patients treated with TARE is evaluated with cross-sectional abdominal imaging, commonly computed tomography (CT) or magnetic resonance imaging (MRI). There is no standard protocol for the timing of postprocedure imaging. At the authors' institution, follow-up imaging with either CT or MRI is obtained 1 month after the procedure, 3 months after the first scan, and every 3-6 months thereafter. Others have proposed that the optimum cross-sectional imaging schedule be $2,4,6,8,11,14,18$, and 24 months postprocedure [28].

During follow-up outpatient visits, patients are assessed for adverse events attributable to TARE, namely abdominal pain, nausea, vomiting, and fatigue. Signs/symptoms of hepatic abscess, perihepatic ascites, pleural effusion, radiation cholecystitis, and radiation pneumonitis (seen in less than $1 \%$ of patients) should be elicited [29]. Nontarget radiation administration may result in gastritis, gastrointestinal ulceration, and other gastrointestinal complications. Patient history and physical examination should be correlated with imaging.

\section{OVERVIEW OF PIVITOL CLINICAL TRIAL DATA}

\section{Overall Survival}

Much of the published literature regarding TARE in HCC has shown consistent results in survival (Table 1). Salem et al. reported an overall survival (OS) of 17.2 months in Child-Pugh A disease [19]. Hilgard et al. achieved a median OS of 16.4 months in 108 patients (47\% BCLC B; 51\% BCLC C) with lobar-directed Y90 [30]. Sangro et al. reported a median OS of 16.9 months in BCLC B patients who were poor candidates for TACE (bilobar disease or $>5$ tumors). In those who failed prior TACE, median OS was 15.4 months [31]. Mazzaferro et al., in the first prospective phase II study of TARE for HCC, obtained a median OS of 15 months-similar to previously reported OS after TACE in BCLC B disease [32].

Many studies in interventional oncology have compared TARE to its transarterial counterpart TACE. No prospective randomized control trials (RCTs) have shown a statistically significant difference in OS between the two therapies. It has been estimated that a study like this may require more than 1000 subjects to reveal an OS difference [33]. Prospective comparator RCTs-such as Transarterial RAdioembolization versus ChemoEmbolization for the treatment of hepatocellular carcinoma (TRACE)-are underway [34].

On the other hand, some retrospective studies have shown statistically significant differences in OS. A recent retrospective study of BCLC B and C HCC patients showed an OS advantage with TARE: mean OS 39 months for 
Table 1 Clinical outcomes of transarterial radioembolization for hepatocellular carcinoma

\begin{tabular}{|c|c|c|c|c|}
\hline & Salem et al. [19] & Hilgard et al. [30] & Sangro et al. [31] & Mazzaferro et al. [32] \\
\hline Patients & 291 TARE pts & 108 TARE pts & 325 TARE pts & $\begin{array}{c}52 \text { TARE pts with } \\
\text { BCLC B and C }\end{array}$ \\
\hline \multirow[t]{2}{*}{ Response rate } & WHO: $42 \%$ & EASL: $40 \%$ & Not assessed & WHO: $40 \%$ \\
\hline & EASL: $57 \%$ & & & EASL: $40 \%$ \\
\hline $\begin{array}{l}\text { Time-to-progression } \\
\text { (months) }\end{array}$ & 7.9 & 10 & Not assessed & 11 \\
\hline \multirow[t]{3}{*}{ Survival (months) } & $\mathrm{CP}^{\mathrm{a}} \mathrm{A}: 17.2$ & 16.4 & BCLC $^{\text {b }}$ A: 24.4 & 15 \\
\hline & CP B: 7.7 & & BCLC B: 16.9 & \\
\hline & & & BCLC C: 10.0 & \\
\hline
\end{tabular}

$B C L C$ Barcelona Clinical Liver Cancer, $C P$ Child-Pugh, pts patients

${ }^{a}$ WHO: World Health Organization criteria that assesses tumor response on cross-sectional imaging based on size of the largest diameter of the tumor

b EASL: European Association for the Study of the Liver criteria that assess tumor response on cross-sectional imaging based on the degree of necrosis

TARE versus 31 months for TACE $(p=0.014)$ [35]. A 2015 meta-analysis of 1499 patients with HCC from 2009 to 2014 also yielded an OS advantage in favor of TARE [hazard ratio $(\mathrm{HR})=0.74 ; \quad 95 \% \quad$ CI $0.61-0.90] \quad$ [36]. In contrast, one study showed that TACE extends a higher OS (15 versus 6 months, $p<0.0001$ ) [37].

\section{Time to Progression}

Though prospective studies have not shown differences in OS, studies have demonstrated improvements in other endpoints of HCC treatment. TARE results in a longer TTP than TACE (13.3 versus 8.4 months, $p=0.046$ ), thus better serving as a bridge to transplant at centers with a long wait time [33]. Hilgard et al. reported a similar TTP of 10 months after TARE [30]. Lewandowski et al. showed that event-free survival, which correlates with TTP, was significantly greater for TARE than TACE
(17.7 versus 7.1 months, $p=0.0017$ ) [38]. A 2015 meta-analysis showed improved TTP with TARE $(H R=0.61 ; 95 \%$ CI 0.41-0.89) [36].

\section{Safety Profile}

TARE is a well-tolerated outpatient therapy, while TACE typically involves brief hospitalization for symptom management. In 2011, Salem et al. published a comparative analysis of patients with intermediate stage HCC treated with TACE $(n=122)$ and TARE $(n=123)$. More patients treated with TACE experienced abdominal pain and transaminitis $(p<0.05)$ [33]. Hilgard et al. reported a more tolerable safety profile with TARE [30]. TARE results in shorter hospitalization time (mean difference of 2.66 days; 95\% CI 4.08-1.24) [36]. El Fouly et al. showed that TARE was better tolerated than TACE, required less hospitalization, and necessitated fewer treatment sessions [39]. Other studies have 
also shown fewer adverse events with TARE [40-43].

\section{Quality of Life}

A prospective study of 29 patients treated with TARE versus 27 patients treated with TACE demonstrated that TARE results in a statistically significant improvement in social well-being, functional well-being, and embolotherapy-specific factors based on the validated Functional Assessment of Cancer Therapy-Hepatobiliary (FACT-Hep) survey. Patients also had a trend toward improved overall quality of life (QoL) [44]. A randomized study showed that a single session of TARE was as safe as multiple sessions of TACE and had a similar impact on health-related QoL [45].

\section{Downstaging Patients to Transplant}

TARE has been shown to downstage over half of patients outside of transplant criteria from United Network of Organ Sharing (UNOS) stage T3 to T2, thus allowing them to undergo transplant [17]. In a comparison study of the two intra-arterial therapies (TACE, $n=43$; TARE, $n=43$ ), TARE was more effective in downstaging patients from UNOS T3 to T2. UNOS T2 was achieved in 31\% of TACE and $58 \%$ of TARE patients [38]. This was also shown in cirrhotic patients with intermediate, advanced, or not-otherwise-treatable HCC [46]. Successful tumor downstaging attributable to TARE has been demonstrated in many other studies [17, 38, 47]. The recurrence-free survival and OS after transplant in the downstaged patients have yet to be compared with those of the patients who were already within transplant criteria.

\section{Portal Vein Thrombosis/Advanced Stage HCC}

Oral sorafenib chemotherapy is considered the standard of care for advanced stage HCC on the basis of the 2008 Sorafenib Hepatocellular carcinoma Assessment Randomized Protocol (SHARP) trial [48]. Emerging evidence has demonstrated TARE to be a viable alternative to sorafenib.

TARE results in similar survival outcomes to sorafenib in the treatment of HCC complicated by PVT, a form of advanced stage HCC. Mazzaferro et al. demonstrated the safety and feasibility of TARE in the setting of PVT. Patients with PVT invading the right or left portal vein or its segmental branches had a median OS of 17 after TARE; patients with main PVT had a 9-month OS [32]. This is comparable to the median OS of 11 months (range 10-13.8 months) reported in previous studies of TARE for advanced HCC $[19,30,31,33,49]$. The median TTP in PVT patients was 13 months in the Mazzaferro cohort [32], confirming the results of the previous studies [19, 30, 31, 33, 49]. Gramenzi et al. has also shown similar OS between TARE and sorafenib (13.1 versus 11.2 months), though only TARE could successfully downstage patients [46].

Some studies have suggested that TARE is more advantageous than sorafenib in PVT. Edeline et al. calculated a median OS of 26.2 versus 8.7 months in patients treated with TARE versus sorafenib, respectively $(p=0.054)$ [50]. Kulik et al. showed that TARE alone was just as effective as TARE combined with sorafenib, although the latter group experienced more biliary complications necessitating reduced sorafenib dose [51]. 


\section{Cost-effectiveness}

Cost analyses investigating the intra-arterial therapies for HCC have been performed. TACE was estimated at US\$17,000; unilobar and bilobar TARE reached US\$31,000 and US $\$ 48,000$, respectively. The incremental cost-effectiveness ratio in the BCLC C subgroup favored TARE by US $\$ 360$ per month. Cost-effectiveness was incomparable in the BCLC B group [52]. Another similar simulated analysis showed that Y90 was less expensive than TACE one-third of the time, although this depends on the need for repeat procedures. Though meticulous, this and other studies did not factor improvement in QoL, days lost from work after treatment, hospital length-of-stay, a higher average number of TACE sessions per patient compared to TARE, and other ancillary costs [53].

\section{NEW DEVELOPMENTS IN THE PRACTICE AND ROLE OF TARE}

\section{Radiation Segmentectomy: Alternative to Ablation}

Although radiofrequency ablation is an adopted standard for early-stage HCC (BCLC A), collateral damage may occur when the lesion is adjacent to critical structures. Highly selective radioembolization (termed radiation segmentectomy) is a feasible alternative to ablation. Riaz et al. demonstrated the safety and efficacy of radiation segmentectomy in the first proof-of-concept study. Positive response to segmental Y90 on imaging occurred in 59-81\% of patients. Median TTP was 13.6 months; median OS was 27 months [54]. Vouche et al. further corroborated this result. After radiation segmentectomy, complete and partial radiologic tumor response was observed in 47/99 (47\%) and 39/99 (39\%), respectively. Pathology in 33 patients who underwent resection revealed $52 \%$ of patients with complete necrosis, all with greater than 90\% necrosis [55]. Padia et al. similarly noted 19 of 20 patients with $100 \%$ tumor response [56].

\section{Radiation Lobectomy: Beyond the Target Tumor}

Some patients are unable to undergo surgical resection of HCC because of inadequate resultant normal liver parenchyma (termed future liver remnant, FLR). This has given rise to neoadjuvant techniques to increase the FLR volume. Currently, portal vein embolization (PVE) is the established method to increase the FLR volume (by redirecting portal venous flow and growth factors to the contralateral lobe of the liver). Achieving an FLR of $40 \%$ prior to resection is an accepted target for patients with cirrhosis (commonly co-presenting with HCC) [57-59]. Unfortunately, PVE may also result in accelerated progression of the untreated tumor as a result of delayed surgery [60].

Radiation lobectomy (RL), the lobar infusion of Y90 with the intent to facilitate surgical resection in patients with small FLR, has been shown to induce volumetric changes comparable to PVE [61-63]. Additionally, RL may be favorable to PVE by providing hepatic tumor control during the time to hypertrophy/ resection. One disadvantage of $\mathrm{RL}$ is the fact that PVE results in faster time to hypertrophy (62.5 versus $29 \%$ at 1 month) [64]. Nevertheless, this is superseded by the fact that PVE may result in acceleration tumor progression, even passing the threshold for resection [65]. Although RL induces contralateral 
hypertrophy in a longer time frame, it confers ipsilateral tumor control, making it a more suitable modality for select patients [66].

\section{Boosted Selective Internal Radiation Therapy (B-SIRT)}

The concept of TARE is to deliver a tumoricidal radiation dose while sparing healthy liver tissue. It has been demonstrated that treatment intensification may incite a better response for more aggressive tumors with portal vein invasion (a concept termed B-SIRT). B-SIRT is also particularly useful for tumors larger than $5 \mathrm{~cm}$. In a 71-patient study, 17 patients received a boosted dosage increased to a target of greater than $205 \mathrm{~Gy}$. The response rate was $76 \%$ versus $9.2 \%$ in boosted versus non-boosted patients, respectively [67]. Although still in a preliminary phase, B-SIRT is showing great promise for providing higher therapeutic doses for complicated HCC. In addition, B-SIRT can serve to tailor the Y90 treatment regimen and personalize the dose delivered to the tumor.

\section{Y90-PET Imaging for Post-TARE Assessment}

Y90-positron emission tomography (PET) imaging is becoming a recognized method to quantify the results of TARE. Y90 undergoes internal pair production when it decays to the stable element zirconium. This decay property of Y90 lends itself to quantitative monitoring via PET scan without the fluorodeoxyglucose (FDG) marker (non-FDG PET). Gates et al., in the first proof-of-concept study, demonstrated a correlation between the Y90-PET signal immediately after TARE and response on 1-month follow-up CT in five patients (three with HCC, two with hepatic metastases) [68]. A recently published study showed that Y90-PET dose-volume histograms correlate with response of hepatic metastases treated with TARE [69]. Another group showed a statistically significant association between absorbed Y90 dose to normal liver and the presence of two or more severe complications after TARE $(p=0.036)$, as well as a possible dose-response trend [70]. Different protocols based on the size of the treated tumor are being investigated [71]. Y90-PET is still investigatory but is showing promise in correlating radiation dose to outcomes, quantifying the response of hepatic tumors to Y90, and ultimately in personalizing dosimetry.

\section{ONGOING CLINICAL TRIALS}

A number of clinical trials investigating TARE in patients with advanced stage HCC are currently underway. The Sorafenib and Micro-therapy Guided by Primovist Enhanced MRI in Patients With Inoperable Liver Cancer (SORAMIC) study evaluates sorafenib \pm sequential Y90 resin therapy. A safety analysis of the first 40 randomized patients (20 from each cohort) has been published revealing similar toxicity at a median follow-up of 8.3 months and median Y90 dosage of $1.87 \mathrm{GBq}$ injected at the lobar level [72]. SorAfenib versus Radioembolisation in Advanced Hepatocellular carcinoma (SARAH) is the first prospective head-to-head RCT of resin microspheres versus sorafenib. The primary endpoint is OS. Secondary endpoints include adverse events rate, progression-free survival (PFS) at 6 months measured by imaging, tumor response rate, QoL, and cost of each strategy [73]. The study to compare Selective Internal Radiation Therapy (SIRT) versus SorafeNIB in Locally Advanced Hepatocellular Carcinoma (HCC) (SIRveNIB) is 
a Singapore-based phase III head-to-head RCT of sorafenib versus resin microspheres. Its primary outcome measure is OS. Secondary measures include overall PFS, PFS in the liver, tumor response rate, safety, QoL, liver resection rate, transplant rate, TTP, and disease control rate. The study was scheduled to stop enrolling by July 2015, but is still currently recruiting participants [74].

There are two currently enrolling studies of glass microsphere radioembolization for advanced stage HCC. The 90Yttrium transarterial radio-Embolization versus Standard of care (sorafenib) for the treatment of advanced hepatocellular carcinoma with Portal vein thrombosis (YES-P) is a head-to-head prospective RCT in which patients either receive Y90 or sorafenib. Its primary endpoint is OS. Secondary endpoints include TTP, time to symptomatic progression, time of worsening PVT, tumor response, patient-reported outcomes, and safety. Another currently enrolling RCT is the Phase III Clinical Trial of Intra-arterial TheraSphere ${ }^{\circledR}$ in the Treatment of Patients with Unresectable Hepatocellular Carcinoma (STOP-HCC) studying sorafenib \pm Y90. Its primary endpoint is OS, with secondary endpoints including TTP, time to untreatable/ symptomatic progression, tumor response, QoL, and safety [75].

\section{CONCLUSION}

TARE with $\mathrm{Y} 90$ is a transarterial procedure that directly delivers radioactive microspheres to tumors to induce cytotoxic injury. In the USA, glass microspheres are indicated for patients with inoperable HCC. All patients undergoing TARE require a history and physical examination, clinical laboratory tests, imaging, and arteriography/ macroaggregated albumin (MAA) lung shunting study. Follow-up imaging with either CT or MRI should be regularly obtained to assess for tumor response and adverse events after the procedure. TARE has been shown in many clinical trials to be safe and effective in the management of unresectable HCC. TARE has many additional roles, including as an alternative to TACE, ablation, and sorafenib chemotherapy, in inducing liver hypertrophy, and in downstaging to liver transplantation. Randomized studies are ongoing, specifically in comparison with sorafenib in the advanced setting, to better define the relative role of TARE in relation to sorafenib. The utility of TARE continues to expand with new developments in interventional oncology.

\section{ACKNOWLEDGMENTS}

No funding or sponsorship was received for this study or publication of this article. All named authors meet the International Committee of Medical Journal Editors (ICMJE) criteria for authorship for this manuscript, take responsibility for the integrity of the work as a whole, and have given final approval for the version to be published.

Disclosures. Robert J. Lewandowski and Riad Salem are advisors to BTG International. Joseph Ralph Kallini and Ahmed Gabr have nothing to disclose.

Compliance with Ethics Guidelines. This article does not contain any studies with human participants or animals performed by any of the authors. Institutional review board 
approval was obtained for cross-sectional imaging included in this review. For this type of study, formal consent is not required.

Open Access. This article is distributed under the terms of the Creative Commons Attribution-NonCommercial 4.0 International License (http://creativecommons.org/licenses/ by-nc/4.0/), which permits any noncommercial use, distribution, and reproduction in any medium, provided you give appropriate credit to the original author(s) and the source, provide a link to the Creative Commons license, and indicate if changes were made.

\section{REFERENCES}

1. Jemal A, Bray F, Center MM, Ferlay J, Ward E, Forman D. Global cancer statistics. CA Cancer J Clin. 2011;61(2):69-90.

2. Carr BI. Tumors of the liver and biliary tree. In: Kasper D, Fauci A, Hauser S, Longo D, Jameson JL, Loscalzo J, editors. Harrison's principles of internal medicine, 19e. New York: McGraw-Hill; 2015.

3. European Association for the Study of the Liver, European Organisation for Research and Treatment of Cancer. EASL-EORTC clinical practice guidelines: management of hepatocellular carcinoma. J Hepatol. 2012;56(4):908-43.

4. Llovet JM, Real MI, Montana X, et al. Arterial embolisation or chemoembolisation versus symptomatic treatment in patients with unresectable hepatocellular carcinoma: a randomised controlled trial. Lancet. 2002;359 (9319):1734-9.

5. Lo CM, Ngan $\mathrm{H}$, Tso $\mathrm{WK}$, et al. Randomized controlled trial of transarterial lipiodol chemoembolization for unresectable hepatocellular carcinoma. Hepatology. 2002;35(5):1164-71.

6. Kallini JR, Miller FH, Gabr A, Salem R, Lewandowski RJ. Hepatic imaging following intra-arterial embolotherapy. Abdom Radiol (NY). 2016. doi:10. 1007/s00261-016-0639-5.

7. Sangro B, Salem R. Transarterial chemoembolization and radioembolization. Semin Liver Dis. 2014;34(4):435-43.
8. National Comprehensive Cancer Network. Hepatobiliary Cancers (Version 1.2016). http:// www.nccn.org/professionals/physician_gls/pdf/hep atobiliary.pdf. Accessed 20 Mar 2016.

9. Galun D, Basaric D, Zuvela M, et al. Hepatocellular carcinoma: From clinical practice to evidence-based treatment protocols. World J Hepatol. 2015;7(20):2274-91. doi:10.4254/wjh.v7.i20.2274.

10. Lewandowski RJ, Salem R. Yttrium-90 radioembolization of hepatocellular carcinoma and metastatic disease to the liver. Semin Intervent Radiol. 2006;23(1):64-72.

11. Hickey R, Mulcahy MF, Lewandowski RJ, et al. Chemoradiation of hepatic malignancies: prospective, phase 1 study of full-dose capecitabine with escalating doses of yttrium-90 radioembolization. Int $\mathrm{J}$ Radiat Oncol Biol Phys. 2014;88(5):1025-31.

12. Kennedy A, Nag $\mathrm{S}$, Salem $\mathrm{R}$, et al. Recommendations for radioembolization of hepatic malignancies using yttrium-90 microsphere brachytherapy: a consensus panel report from the radioembolization brachytherapy oncology consortium. Int J Radiat Oncol Biol Phys. 2007;68(1):13-23.

13. Carr BI. Hepatic arterial 90Yttrium glass microspheres (Therasphere) for unresectable hepatocellular carcinoma: interim safety and survival data on 65 patients. Liver Transplant. 2004;10(2 Suppl 1):S107-10.

14. Dancey JE, Shepherd FA, Paul K, et al. Treatment of nonresectable hepatocellular carcinoma with intrahepatic 90Y-microspheres. J Nucl Med. 2000;41(10):1673-81.

15. Geschwind JF, Salem R, Carr BI, et al. Yttrium-90 microspheres for the treatment of hepatocellular carcinoma. Gastroenterology. 2004;127(5 Suppl 1):S194-205.

16. Goin J, Roberts C, Dancey J, Sickles C, Leung D, Soulen M. Comparison of post-embolization syndrome in the treatment of patients with unresectable hepatocellular carcinoma: trans-catheter arterial chemo-embolization versus yttrium-90 glass microspheres. World J Nucl Med. 2004;3(1):49-56.

17. Kulik LM, Atassi B, van Holsbeeck L, et al. Yttrium-90 microspheres (TheraSphere) treatment of unresectable hepatocellular carcinoma: downstaging to resection, RFA and bridge to transplantation. J Surg Oncol. 2006;94(7):572-86.

18. Lau WY, Ho S, Leung TW, et al. Selective internal radiation therapy for nonresectable hepatocellular 
carcinoma with intraarterial infusion of 90yttrium microspheres. Int $\mathrm{J}$ Radiat Oncol Biol Phys. 1998;40(3):583-92.

19. Salem R, Lewandowski RJ, Mulcahy MF, et al. Radioembolization for hepatocellular carcinoma using yttrium-90 microspheres: a comprehensive report of long-term outcomes. Gastroenterology. 2010;138(1):52-64.

20. Sangro B, Carpanese L, Cianni R, et al. Survival after yttrium-90 resin microsphere radioembolization of hepatocellular carcinoma across Barcelona clinic liver cancer stages: a European evaluation. Hepatology. 2011;54(3):868-78.

21. Gates VL, Marshall KG, Salzig K, Williams M, Lewandowski RJ, Salem R. Outpatient single-session yttrium-90 glass microsphere radioembolization. J Vasc Intervent Radiol. 2014;25(2):266-70.

22. TheraSphere Yttrium-90 microspheres package insert. Kanata CMN. http://www.therasphere.com/ physicians-package-insert/TS_PackageInsert_USA_ v12.pdf.

23. SIRS-Spheres Yttrium-90 microspheres package insert. Singapore Science Park SSM. http://www. sirtex.com/media/29845/ssl-us-10.pdf.

24. Piana PM, Bar V, Doyle L, et al. Early arterial stasis during resin-based yttrium-90 radioembolization: incidence and preliminary outcomes. HPB (Oxford). 2014;16(4):336-41.

25. Liu DM, Salem R, Bui JT, et al. Angiographic considerations in patients undergoing liver-directed therapy. J Vasc Intervent Radiol. 2005;16(7):911-35.

26. Hamoui N, Minocha J, Memon $\mathrm{K}$, et al. Prophylactic embolization of the gastroduodenal and right gastric arteries is not routinely necessary before radioembolization with glass microspheres. J Vasc Intervent Radiol. 2013;24(11):1743-5.

27. Salem R, Parikh P, Atassi B, et al. Incidence of radiation pneumonitis after hepatic intra-arterial radiotherapy with yttrium-90 microspheres assuming uniform lung distribution. Am J Clin Oncol. 2008;31(5):431-8.

28. Boas FE, Do B, Louie JD, et al. Optimal imaging surveillance schedules after liver-directed therapy for hepatocellular carcinoma. J Vasc Intervent Radiol. 2015;26(1):69-73.

29. Miller FH, Keppke AL, Reddy D, et al. Response of liver metastases after treatment with yttrium-90 microspheres: role of size, necrosis, and PET. AJR Am J Roentgenol. 2007;188(3):776-83.
30. Hilgard P, Hamami M, Fouly AE, et al. Radioembolization with yttrium-90 glass microspheres in hepatocellular carcinoma: European experience on safety and long-term survival. Hepatology. 2010;52(5):1741-9.

31. Sangro B, Carpanese L, Cianni R, et al. Survival after yttrium-90 resin microsphere radioembolization of hepatocellular carcinoma across Barcelona clinic liver cancer stages: a European evaluation. Hepatology. 2011;54(3):868-78.

32. Mazzaferro V, Sposito C, Bhoori S, et al. Yttrium-90 radioembolization for intermediate-advanced hepatocellular carcinoma: a phase 2 study. Hepatology. 2013;57(5):1826-37.

33. Salem R, Lewandowski RJ, Kulik L, et al. Radioembolization results in longer time-to-progression and reduced toxicity compared with chemoembolization in patients with hepatocellular carcinoma. Gastroenterology. 2011;140(2):497-507.e2.

34. Seinstra BA, Defreyne L, Lambert B, et al. Transarterial radioembolization versus chemoembolization for the treatment of hepatocellular carcinoma (TRACE): study protocol for a randomized controlled trial. Trials. 2012;13:144.

35. Soydal C, Arslan MF, Kucuk ON, Idilman R, Bilgic S. Comparison of survival, safety, and efficacy after transarterial chemoembolization and radioembolization of Barcelona Clinic Liver Cancer stage B-C hepatocellular cancer patients. Nucl Med Commun. 2016 (in press).

36. Zhang Y, Li Y, Ji H, Zhao X, Lu H. Transarterial Y90 radioembolization versus chemoembolization for patients with hepatocellular carcinoma: a meta-analysis. Biosci Trends. 2015;9(5):289-98.

37. Akinwande O, Philips P, Scoggins C, Martin RC. Radioembolization versus chemoembolization (DEBDOX) for the treatment of unresectable hepatocellular carcinoma: a propensity matched study. Anticancer Res. 2016;36(1):239-46.

38. Lewandowski RJ, Kulik LM, Riaz A, et al. A comparative analysis of transarterial downstaging for hepatocellular carcinoma: chemoembolization versus radioembolization. Am J Transplant. 2009;9(8):1920-8.

39. El Fouly A, Ertle J, El Dorry A, et al. In intermediate stage hepatocellular carcinoma: radioembolization with yttrium 90 or chemoembolization? Liver Int. 2015;35(2):627-35.

40. Xing M, Kokabi N, Camacho JC, Kooby DA, El-Rayes BF, Kim HS. 90Y radioembolization versus 
chemoembolization in the treatment of hepatocellular carcinoma: an analysis of comparative effectiveness. J Comp Eff Res. 2013;2(4):435-44.

41. Moreno-Luna LE, Yang JD, Sanchez W, et al. Efficacy and safety of transarterial radioembolization versus chemoembolization in patients with hepatocellular carcinoma. Cardiovasc Intervent Radiol. 2013;36(3):714-23.

42. Lance C, McLennan G, Obuchowski N, et al. Comparative analysis of the safety and efficacy of transcatheter arterial chemoembolization and yttrium-90 radioembolization in patients with unresectable hepatocellular carcinoma. J Vasc Intervent Radiol. 2011;22(12):1697-705.

43. Kooby DA, Egnatashvili V, Srinivasan S, et al. Comparison of yttrium-90 radioembolization and transcatheter arterial chemoembolization for the treatment of unresectable hepatocellular carcinoma. J Vasc Intervent Radiol. 2010;21(2):224-30.

44. Salem R, Gilbertsen M, Butt Z, Memon K, Vouche $\mathrm{M}$, Hickey $\mathrm{R}$, et al. Increased quality of life among hepatocellular carcinoma patients treated with radioembolization, compared with chemoembolization. Clin Gastroenterol Hepatol. 2013;11(10):1358-65.e1.

45. Kolligs FT, Bilbao JI, Jakobs $\mathrm{T}$, et al. Pilot randomized trial of selective internal radiation therapy vs. chemoembolization in unresectable hepatocellular carcinoma. Liver Int. 2015;35(6):1715-21.

46. Gramenzi A, Golfieri R, Mosconi C, et al. Yttrium-90 radioembolization vs sorafenib for intermediate-locally advanced hepatocellular carcinoma: a cohort study with propensity score analysis. Liver Int. 2015;35(3):1036-47.

47. Ibrahim SM, Kulik L, Baker T, et al. Treating and downstaging hepatocellular carcinoma in the caudate lobe with yttrium-90 radioembolization. Cardiovasc Intervent Radiol. 2012;35(5):1094-101.

48. Llovet JM, Ricci S, Mazzaferro V, et al. Sorafenib in advanced hepatocellular carcinoma. N Engl J Med. 2008;359(4):378-90.

49. Inarrairaegui $\mathrm{M}$, Thurston $\mathrm{KG}$, Bilbao JI, et al. Radioembolization with use of yttrium-90 resin microspheres in patients with hepatocellular carcinoma and portal vein thrombosis. J Vasc Intervent Radiol. 2010;21(8):1205-12.

50. Edeline J, Crouzet L, Campillo-Gimenez B, et al. Selective internal radiation therapy compared with sorafenib for hepatocellular carcinoma with portal vein thrombosis. Eur J Nucl Med Mol Imaging. 2016;43(4):635-43.

51. Kulik L, Vouche M, Koppe S, et al. Prospective randomized pilot study of Y90 \pm sorafenib as bridge to transplantation in hepatocellular carcinoma. J Hepatol. 2014;61(2):309-17.

52. Rostambeigi N, Dekarske AS, Austin EE, Golzarian J, Cressman EN. Cost effectiveness of radioembolization compared with conventional transarterial chemoembolization for treatment of hepatocellular carcinoma. J Vasc Intervent Radiol. 2014;25(7):1075-84.

53. Ray CE Jr, Battaglia C, Libby AM, Prochazka A, Xu S, Funaki B. Interventional radiologic treatment of hepatocellular carcinoma-a cost analysis from the payer perspective. J Vasc Intervent Radiol. 2012;23(3):306-14.

54. Riaz A, Gates VL, Atassi B, et al. Radiation segmentectomy: a novel approach to increase safety and efficacy of radioembolization. Int $\mathrm{J}$ Radiat Oncol Biol Phys. 2011;79(1):163-71.

55. Vouche M, Habib A, Ward TJ, et al. Unresectable solitary hepatocellular carcinoma not amenable to radiofrequency ablation: multicenter radiology-pathology correlation and survival of radiation segmentectomy. Hepatology. 2014;60(1):192-201.

56. Padia SA, Kwan SW, Roudsari B, Monsky WL, Coveler A, Harris WP. Superselective yttrium-90 radioembolization for hepatocellular carcinoma yields high response rates with minimal toxicity. J Vasc Intervent Radiol. 2014;25(7):1067-73.

57. Kishi Y, Abdalla EK, Chun YS, et al. Three hundred and one consecutive extended right hepatectomies: evaluation of outcome based on systematic liver volumetry. Ann Surg. 2009;250(4):540-8.

58. Farges O, Belghiti J, Kianmanesh R, et al. Portal vein embolization before right hepatectomy: prospective clinical trial. Ann Surg. 2003;237(2):208-17.

59. van Lienden KP, van den Esschert JW, de Graaf W, et al. Portal vein embolization before liver resection: a systematic review. Cardiovasc Intervent Radiol. 2013;36(1):25-34.

60. de Graaf W, van den Esschert JW, van Lienden KP, van Gulik TM. Induction of tumor growth after preoperative portal vein embolization: is it a real problem? Ann Surg Oncol. 2009;16(2):423-30.

61. Gaba RC, Lewandowski RJ, Kulik LM, et al. Radiation lobectomy: preliminary findings of hepatic volumetric response to lobar yttrium-90 
radioembolization. Ann Surg Oncol. 2009;16(6):1587-96.

62. Edeline J, Lenoir L, Boudjema K, et al. Volumetric changes after $(90) \mathrm{Y}$ radioembolization for hepatocellular carcinoma in cirrhosis: an option to portal vein embolization in a preoperative setting? Ann Surg Oncol. 2013;20(8):2518-25.

63. Ahmadzadehfar H, Meyer C, Ezziddin S, et al. Hepatic volume changes induced by radioembolization with $90 \mathrm{Y}$ resin microspheres. A single-centre study. Eur J Nucl Med Mol Imaging. 2013;40(1):80-90.

64. Vouche M, Lewandowski RJ, Atassi R, et al. Radiation lobectomy: time-dependent analysis of future liver remnant volume in unresectable liver cancer as a bridge to resection. J Hepatol. 2013;59(5):1029-36.

65. Pamecha V, Glantzounis G, Davies N, Fusai G, Sharma D, Davidson B. Long-term survival and disease recurrence following portal vein embolisation prior to major hepatectomy for colorectal metastases. Ann Surg Oncol. 2009;16(5):1202-7.

66. Garlipp B, de Baere T, Damm R, et al. Left-liver hypertrophy after therapeutic right-liver radioembolization is substantial but less than after portal vein embolization. Hepatology. 2014;59(5):1864-73.

67. Garin E, Lenoir L, Edeline J, et al. Boosted selective internal radiation therapy with 90Y-loaded glass microspheres (B-SIRT) for hepatocellular carcinoma patients: a new personalized promising concept. Eur J Nucl Med Mol Imaging. 2013;40(7):1057-68.

68. Gates VL, Esmail AA, Marshall K, Spies S, Salem R. Internal pair production of $90 \mathrm{Y}$ permits hepatic localization of microspheres using routine PET: proof of concept. J Nucl Med. 2011;52(1):72-6.

69. Fowler KJ, Maughan NM, Laforest R, et al. PET/MRI of hepatic $90 \mathrm{Y}$ microsphere deposition determines individual tumor response. Cardiovasc Intervent Radiol. 2015. doi:10.1007/s00270-015-1285-y.

70. Srinivas SM, Natarajan N, Kuroiwa J, et al. Determination of radiation absorbed dose to primary liver tumors and normal liver tissue using post-radioembolization (90)Y PET. Front Oncol. $2014 ; 4: 255$.

71. Attarwala AA, Molina-Duran F, Busing KA, et al. Quantitative and qualitative assessment of yttrium-90 PET/CT imaging. PLoS One. 2014;9(11):e110401.

72. Ricke J, Bulla K, Kolligs F, et al. Safety and toxicity of radioembolization plus sorafenib in advanced hepatocellular carcinoma: analysis of the European multicentre trial SORAMIC. Liver Int. 2015;35(2):620-6.

73. Vilgrain V, Abdel-Rehim $\mathrm{M}$, Sibert A, et al. Radioembolisation with yttrium90 microspheres versus sorafenib for treatment of advanced hepatocellular carcinoma (SARAH): study protocol for a randomised controlled trial. Trials. 2014;15:474.

74. Clinicaltrials.gov. Study to compare selective internal radiation therapy (SIRT) versus sorafenib in locally advanced hepatocellular carcinoma (HCC) (SIRveNIB). Updated 26 Nov 2014. https:// clinicaltrials.gov/ct2/show/study/NCT01135056? term $=01135056 \&$ rank $=1$. Accessed cited $17 \mathrm{Mar}$ 2016.

75. Clinical Trials With TheraSphere ${ }^{\circledR}$ YTTRIUM-90 GLASS MICROSPHERES. 2016. http://www. therasphere.com/clinicaltrials_us/index.asp. 